\title{
SOLUTION OF VOLTERRA-TYPE INTEGRO-DIFFERENTIAL EQUATIONS WITH A GENERALIZED LAURICELLA CONFLUENT HYPERGEOMETRIC FUNCTION IN THE KERNELS
}

\author{
R. K. SAXENA AND S. L. KALLA
}

Received 1 March 2005

The object of this paper is to solve a fractional integro-differential equation involving a generalized Lauricella confluent hypergeometric function in several complex variables and the free term contains a continuous function $f(\tau)$. The method is based on certain properties of fractional calculus and the classical Laplace transform. A Cauchy-type problem involving the Caputo fractional derivatives and a generalized Volterra integral equation are also considered. Several special cases are mentioned. A number of results given recently by various authors follow as particular cases of formulas established here.

\section{Introduction and preliminaries}

The first-order integro-differential equation of Volterra type [7, 9]

$$
\frac{d}{d \tau} a(\tau)=-i \pi g_{0} \int_{0}^{\tau} \varsigma a(\tau-\varsigma) \exp (i \nu \varsigma) d \varsigma
$$

describes the unsaturated behavior of the free electron laser (FEL). Here $\tau$ is a dimensionless time variable, $g_{0}$ is a positive constant called the small signal gain, and the detuning parameter is the constant $\nu$. The function $a(\tau)$ is the complex-field amplitude which is assumed to be dimensionless satisfying the initial condition $a(0)=1$. The exact closed form solution of (1.1) valid in the whole range of practical interest and suitable for numerical calculations was given by Dattoli et al. [8].

Fractional calculus has gained importance during the last three decades or so due to its various applications in the solution of fractional differential and fractional integral equations arising in various problems of physics engineering and applied sciences, such as diffusion in porous media, fractal geometry, kinematics in viscoelastic media, propagation of seismic waves, anomalous diffusion, and so forth. In this connection, one can refer to the works mentioned in $[6,12,16,18,20,21,25]$.

The Riemann-Liouville operator of fractional integration of order $v$ is defined by

$$
D_{x}^{-\nu}[h(x)]=\frac{1}{\Gamma(\nu)} \int_{0}^{x}(x-t)^{\nu-1} h(t) d t \quad(\operatorname{Re}(\nu)>0),
$$

provided that the integral (1.2) exists. 
The Riemann-Liouville fractional derivative of order $v$ is defined in the form $[19,20$, $21,25]$

$$
D_{x}^{\alpha}[h(x)]=\frac{1}{\Gamma(n-\alpha)} \frac{d^{n}}{d t^{n}} \int_{0}^{t} \frac{h(u) d u}{(t-u)^{\alpha-n+1}} \quad(n-1<\alpha \leq n) .
$$

By the application of the convolution theorem of the Laplace transform [11, page 131], we find from (1.2) that

$$
L\left\{D_{t}^{-\nu} h(t) ; s\right\}=L\left\{\frac{t^{\nu-1}}{\Gamma(\nu)}\right\} L\{h(t)\}=s^{-\nu} H(s),
$$

where $H(s)$ is the Laplace transform of $h(t)$ defined by

$$
L\{h(t): s\}=\int_{0}^{\infty} h(t) e^{-s t} d t=: H(s), \quad \operatorname{Re}(s)>0,
$$

which may be written symbolically as follows:

$$
H(s)=L\{h(t): s\} \quad \text { or } \quad h(t)=L^{-1}\{H(s): t\},
$$

provided that the function $h(t)$ is continuous for $t \geq 0$ and of exponential order as $t \rightarrow \infty$.

Boyadjiev et al. [5, (7), page 4] studied the following nonhomogeneous form of fractional integro-differential equation of Volterra type:

$$
D_{\tau}^{\alpha} a(\tau)=\lambda \int_{0}^{\tau} \varsigma a(\tau-\zeta) \exp (i \nu \zeta) d \zeta+\beta \exp (i v \tau), \quad 0 \leq \tau \leq 1,
$$

where $\beta, \lambda \in \mathbb{C}$ and $\nu \in \mathbb{R}$.

Al-Shammery et al. [3, (14), page 82] considered a generalization of (1.4) in the form

$$
D_{\tau}^{\alpha} a(\tau)=\lambda \int_{0}^{\tau} u^{\delta} a(\tau-u) \exp (i v \tau) d u+\beta \exp (i v \tau) \quad(0 \leq \tau \leq 1)
$$

with $\beta, \lambda, \delta \in \mathbb{C}, \nu \in \mathbb{R}, \operatorname{Re}(\alpha)>0$, and $\operatorname{Re}(\delta)>-1$.

Al-Shammery et al. [2, (14), page 503] further studied another generalization of (1.7) in the form

$$
D_{\tau}^{\alpha} a(\tau)=\lambda \int_{0}^{\tau} u^{\delta} a(\tau-u) \Phi(b, \delta+1 ; i \nu u) d u+\beta \Phi\left(b^{\prime}, 1 ; i v \tau\right)
$$

where $0 \leq \tau \leq 1, \alpha, \beta, \lambda, \delta \in \mathbb{C} ; b, b^{\prime} \in \mathbb{R}, \operatorname{Re}(\alpha)>0, \operatorname{Re}(\delta)>0$, and $\Phi(a, b ; z)$ is the Kummer confluent hypergeometric function defined in [10, (1), page 248].

Saxena and Kalla [26] derived the solution of a further generalizaion of (1.9) in the form

$$
D_{\tau}^{\alpha} h(\tau) \lambda \int_{0}^{\infty} t^{\delta} h(\tau-t) \Phi(b, \delta+1 ; i v u) d u+\mu \tau^{\gamma} \Phi(\beta, \gamma+1 ; i v \tau)
$$

where $0 \leq \tau \leq 1, \alpha, \delta, \lambda, \mu \in \mathbb{C}, \nu, b, \beta \in \mathbb{R}, \operatorname{Re}(\alpha)>0, \operatorname{Re}(\gamma)>-1, \operatorname{Re}(\delta)>-1$. 
Recently Kilbas et al. [13] systematically studied a generalization of (1.10) in the following form:

$$
D_{x}^{\alpha} h(x)=\lambda \int_{a}^{x}(x-t)^{\mu-1} E_{\rho, \mu}^{\gamma}\left[\omega(x-t)^{\rho}\right] h(t) d t+f(x)
$$

where $a \leq x \leq b ; \lambda, \mu, \rho, \gamma \in \mathbb{C} ; \omega \in \mathbb{R}, \operatorname{Re}(\alpha)>0, \operatorname{Re}(\mu)>0$, and $f$ is assumed to be Lebesgue integrable over the interval $(a, b)$ and the function $E_{\rho, \mu}^{\gamma}(z)$ is defined by [22]

$$
E_{\rho, \mu}^{\gamma}(z):=\sum_{r=0}^{\infty} \frac{(\gamma)_{t r} z^{r}}{\Gamma(r \rho+\mu)(r !)}
$$

where $\operatorname{Re}(\rho)>0 ;(\gamma)_{n}:=\Gamma(\gamma+n) / \Gamma(\gamma)$ is a generalization of the classical Mittag-Leffler functions $E_{\rho}(z)$ and $E_{\rho, \mu}(z)$. In terms of the $H$-function, we have [14]

$$
E_{\rho, \mu}^{\gamma}(z)=\frac{1}{\Gamma(\gamma)} H_{1,2}^{1,1}\left[Z \mid \begin{array}{c}
(1-\gamma, 1) \\
(0,1),(1-\mu, \rho)
\end{array}\right] .
$$

A comprehensive account of the various generalizations of FEL equations is recently given in a survey paper by Boyadjiev and Kalla [4]. A description of various special functions appearing in this paper is available in $[1,10,11,31]$.

A detailed account of various operators of fractional integration and their applications can be found in a recent survey paper of Srivastava and Saxena [32]. An interesting account of convolution integral equations has been given by Srivastava and Buschman [28].

The main object of the present paper is to introduce a further generalization of (1.11) in an interesting and unified form in which the generalized Mittag-Leffler function, the involved kernel, is replaced by a generalized Lauricella confluent hypergeometric function in several complex variables and the free term contains a continuous function $f(\tau)$. The method followed here in finding the solution of Cauchy-type problem (3.1) and (3.2) and other Cauchy problems is based upon certain properties of fractional calculus and the classical Laplace transform. The solutions derived are in closed forms and are suitable for numerical computation.

\section{Integral representations of generalized Lauricella confluent hypergeometric function of several complex variables}

The following result is quite interesting and useful as it gives the inverse Laplace transforms of the product of binomial functions:

$$
L^{-1}\left\{s^{-\lambda} \prod_{j=1}^{n}\left[1-\frac{\omega_{j}}{s^{\rho_{j}}}\right]^{-\gamma_{j}}\right\}=t^{\lambda-1} F_{1: \underbrace{0 ; \ldots ; 0}_{n}}^{0: 1 ; \ldots ;}\left[\begin{array}{c}
-:\left(\gamma_{1}, 1\right) ; \ldots ;\left(\gamma_{n}, 1\right) ;- \\
\omega_{1} t^{\rho_{1}}, \ldots, \omega_{n} t^{\rho_{n}} \\
\left(\lambda: \rho_{1}, \ldots, \rho_{n}\right):-:-
\end{array}\right],
$$

where $\lambda, s, \gamma_{j}, \rho_{j}, \omega_{j} \in \mathbb{C}, \operatorname{Re}(s)>0, \max _{1 \leq j \leq n}\left|\omega_{j} / s^{\rho_{j}}\right|<1, \min _{1 \leq j \leq m} \operatorname{Re}\left(\rho_{j}\right)>0, \operatorname{Re}(\lambda)>0$. The formula (2.1) can be easily established by expanding each of the binomial functions 
appearing in (2.1) and interpreting it with the help of the formula

$$
L^{-1}\left[s^{-\rho}: t\right]=\frac{t^{\rho-1}}{\Gamma(\rho)}, \quad \min \{\operatorname{Re}(\rho), \operatorname{Re}(s)\}>0,
$$

where the function

$$
F_{n}^{0: 1 ; \ldots ; 1} \underbrace{n}_{n}\left[\begin{array}{c}
-:\left(\gamma_{1}, 1\right) ; \ldots ;\left(\gamma_{n}, 1\right) ;- \\
\omega_{1} t^{\rho_{1}}, \ldots, \omega_{n} t^{\rho_{n}} \\
\left(\gamma: \rho_{1}, \ldots, \rho_{n}\right):-:-
\end{array}\right]
$$

is defined for complex $\lambda, \gamma_{j}, \rho_{j}, \omega_{j}, z_{j}\left(\operatorname{Re}\left(\rho_{j}\right)>0\right) j=1, \ldots, n$, in terms of a multiple series in the following form:

$$
F_{1: 0 ; \ldots ; 0}^{0: \overbrace{1 ; \ldots ; 1}^{n}}\left[\begin{array}{c}
-:\left(\gamma_{1}, 1\right) ; \ldots ;\left(\gamma_{n}, 1\right) ;- \\
z_{1}, \ldots, z_{n} \\
\left(\lambda: \rho_{1}, \ldots, \rho_{n}\right):-:-
\end{array}\right]=\sum_{k_{1}, \ldots, k_{n}=0}^{\infty} \frac{\left(\gamma_{1}\right)_{k_{1}} \cdots\left(\gamma_{n}\right)_{k_{j}} z_{1}^{k_{1}} \cdots z_{n}^{k_{n}}}{\Gamma\left[\lambda+\rho_{1} k_{1}+\cdots+\rho_{n} k_{n}\right]\left(k_{1}\right) ! \cdots\left(k_{n}\right) !},
$$

which is a special case of the generalized Lauricella series in several complex variables, introduced by Srivastava and Daoust [29, page 454]. According to the convergence conditions investigated by Srivastava and Daoust [30, page 157] for the generalized Lauricella series in several variables, the series in $(2.4)$ converges for $\operatorname{Re}\left(\rho_{j}\right)>0$ for $j=1, \ldots, n$. Its Mellin-Barnes type integral representation has been given by Saigo and Saxena [24, page 46] as

$$
\begin{aligned}
& F_{\underbrace{0: 0 ; \ldots ; 0}_{n}}^{\overbrace{1 ; \ldots ;}^{n}}\left[\begin{array}{c}
-:\left(\gamma_{1}, 1\right) ; \ldots ;\left(\gamma_{n}, 1\right) ;- \\
z_{1}, \ldots, z_{n} \\
\left(\lambda: \rho_{1}, \ldots, \rho_{n}\right):-:-
\end{array}\right] \\
& \quad=\frac{1}{(2 \pi i)^{n} \prod_{j=1}^{n} \Gamma\left(\gamma_{j}\right)} \int_{\Omega_{1}} \cdots \int_{\Omega_{n}} \frac{\prod_{j=1}^{n}\left[\left\{\Gamma\left(\gamma_{j}+s_{j}\right) \Gamma\left(-s_{j}\right)\right\}\left\{\left(-z_{j}\right)^{s_{j}}\right\}\right]}{\Gamma\left[\lambda+\rho_{1} s_{1}+\cdots+\rho_{n} s_{n}\right]} d s_{1} \cdots d s_{n} .
\end{aligned}
$$

Here the contours $\Omega_{j}$ 's are given by $\Omega_{j}=\Omega_{i s_{j} \infty}\left(i=(-1)^{1 / 2}, \operatorname{Re}\left(s_{j}\right)=v_{j}\right)$ starting at the point $v_{j}-i \infty$ and terminating at the point $\nu_{j}+i \infty ; v_{j} \in \mathfrak{R}(j=1, \ldots, n)$. All the poles of the gamma functions appearing in the integrand of (2.5) are assumed to be simple.

When $n=1,(2.1)$ reduces to the generalized Mittag-Leffler function due to Prabhakar [22] defined by (1.12), consequently the results obtained in this paper will generalize the work reported earlier by Kilbas et al. [13].

If we take $\rho_{1}=\cdots=\rho_{n}=1$ in $(2.1)$, we arrive at a known result [11, page 222, Art. 4.24, equation (5)], namely, if $\operatorname{Re}(s)>0,\left|\omega_{j} / s\right|<1, \min _{1 \leq j \leq n} \operatorname{Re}\left(\rho_{j}\right)>0, \operatorname{Re}(\lambda)>0, \lambda, \rho_{j}, \gamma_{j}$ $\in \mathbb{C}(j=1, \ldots, n)$,

$$
L^{-1}\left\{s^{-\lambda} \prod_{j=1}^{n}\left(1-\frac{\omega_{j}}{s}\right)^{-\gamma_{j}}\right\}=\frac{1}{\Gamma(\lambda)} t^{\lambda-1} \Phi_{2}^{(n)}\left[\gamma_{1}, \ldots, \gamma_{n} ; \lambda ; \omega_{1} t, \ldots, \omega_{n} t\right]
$$


which is defined as

$$
\begin{aligned}
\Phi_{2}^{(n)}[ & \left.\alpha_{1}, \ldots, \alpha_{n} ; \lambda ; z_{1}, \ldots, z_{n}\right] \\
& =\sum_{k_{1}, \ldots, k_{n}=0}^{\infty} \frac{\left(\alpha_{1}\right)_{k_{1}} \cdots\left(\alpha_{n}\right)_{k_{n}}}{(\lambda)_{k_{1}+\cdots+k_{n}}} \frac{z_{1}^{k_{1}} \cdots z_{k}^{k_{n}}}{\left(k_{1}\right) ! \cdots\left(k_{n}\right) !}
\end{aligned}
$$

$\left(\max \left\{\left|z_{1}\right|, \ldots,\left|z_{n}\right|\right\}<\infty ; \lambda \in Z_{0}^{-}\right)$.

For $n=1,(2.1)$ yields the following result given by Kilbas et al. [14, page 37]; also see [22, page 11]: if $\operatorname{Re}(s)>0, \operatorname{Re}(\lambda)>0, \operatorname{Re}(\rho)>0 ;\left|\omega / s^{\rho}\right|<1 ; \lambda, \rho, \gamma, \omega, s \in \mathbb{C}$, then

$$
L\left[t^{\lambda-1} E_{\rho, \lambda}^{\gamma}\left(\omega t^{\rho}\right): s\right]=s^{-\lambda}\left(1-\frac{\omega}{s^{\rho}}\right)^{-\gamma} .
$$

Remark 2.1. Various practical problems of probability and statistics where $\phi_{2}^{(m)}(\cdot)$ appears naturally are discussed by Mathai and Saxena [17].

By the application of the well-known convolution theorem of the Laplace transform, the following summation formula is obtained for the generalized Lauricella confluent hypergeometric function of " $n$ " complex variables. Let $\lambda, \mu, z_{j}, \rho_{j}, \gamma_{j}, \delta_{j} \in \mathbb{C}, \min \{\operatorname{Re}(\lambda)$, $\operatorname{Re}(\mu)\}>0, \min _{1 \leq j \leq m} \operatorname{Re}\left(\rho_{j}\right)>0, \max _{1 \leq j \leq m}\left\{\left|z_{j} t^{\rho_{j}}\right|\right\}<\infty(j=1, \ldots, n)$, then

$$
\begin{aligned}
& \int_{0}^{x} t^{\lambda-1} F_{1: \underbrace{0: \ldots ; 0 ; 0}_{n}}^{\overbrace{1 ; \ldots ; 1}^{n}}\left[\begin{array}{c}
-:\left(\gamma_{1}: 1\right) ; \ldots ;\left(\gamma n_{n}: 1\right) ;- \\
z_{1} t^{\rho_{1}}, \ldots, z_{n} t^{\rho_{n}} \\
\left(\lambda: \rho_{1}, \ldots, \rho_{n}\right):-:-
\end{array}\right] \\
& \times(x-t)^{\mu-1} F_{1: \underbrace{0: \ldots ; \ldots ; 0}_{n}}^{n}\left[\begin{array}{c}
-:\left(\delta_{1}: 1\right) ; \ldots ;\left(\delta_{n}: 1\right) ;- \\
z_{1}(x-t)^{\rho_{1}}, \ldots, z_{n}(x-t)^{\rho_{n}} \\
\left(\mu: \rho_{1}, \ldots, \rho_{n}\right):-:-
\end{array}\right] d t
\end{aligned}
$$

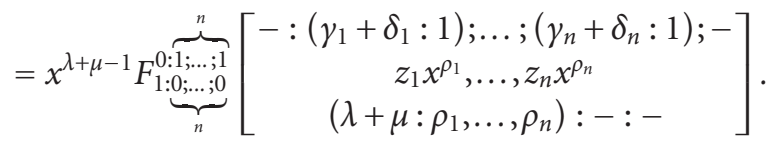

If we set $\delta_{1}=\cdots=\delta_{n}=0$ in (2.9), it yields

$$
\begin{gathered}
\int_{0}^{x} t^{\lambda-1}(x-t)^{\mu-1} \underbrace{n}_{F_{1: 0 ; \ldots ; 0}^{0: 1 ; 0 ; 1}}\left[\begin{array}{c}
-:\left(\gamma_{1}: 1\right) ; \ldots ;\left(\gamma_{n}: 1\right) ;- \\
z_{1}(x-t)^{\rho_{1}}, \ldots, z_{n}(x-t)^{\rho_{n}} \\
\left(\mu: \rho_{1}, \ldots, \rho_{n}\right):-:-
\end{array}\right] d t \\
=x^{\lambda+\mu-1} F_{1: 0 ; \ldots ; 0}^{0: \overbrace{1 ; \ldots ;}^{n}}\left[\begin{array}{c}
-:\left(\gamma_{1}: 1\right) ; \ldots ;\left(\gamma_{n}: 1\right) ;- \\
z_{1} x^{\rho_{1}}, \ldots, z_{n} x^{\rho_{n}} \\
\left(\lambda+\mu: \rho_{1}, \ldots, \rho_{n}\right):-:-
\end{array}\right]
\end{gathered}
$$

which holds under the various conditions stated along with (2.9). If we set $\rho_{1}=\cdots=$ $\rho_{n}=1$, then by virtue of the identity

$$
F_{1: 0 ; 0 ; 0 ; 0}^{0: \overbrace{n}^{n}}\left[\begin{array}{c}
-:\left(\alpha_{1}: 1\right) ; \ldots ;\left(\alpha_{n}: 1\right) ;- \\
z_{1}, \ldots, z_{n} \\
(\lambda: 1, \ldots, 1)
\end{array}\right]=\frac{1}{\Gamma(\lambda)} \Phi_{2}^{(n)}\left[\alpha_{1}, \ldots, \alpha_{n} ; \lambda ; z_{1}, \ldots, z_{n}\right],
$$


the generalized Lauricella confluent hypergeometric function reduces to the Lauricella confluent hypergeometric function $\Phi_{2}^{(n)}(\cdot)$ of " $n$ " variables, and consequently we arrive at the following result recently given by Srivastava and Saxena [33]:

$$
\begin{aligned}
& \int_{0}^{x} t^{\lambda-1}(x-t)^{\mu-1} \Phi_{2}^{(n)}\left[a_{1}, \ldots, a_{n}: \lambda ; \lambda_{1} t, \ldots, \lambda_{n} t\right] \\
& \quad \times \Phi_{2}^{(n)}\left[b_{1}, \ldots, b_{n} ; \mu ; \lambda_{1}(x-t), \ldots, \lambda_{n}(x-t)\right] d t \\
& \quad=B(\lambda, \mu) x^{\lambda+\mu-1} \Phi_{2}^{(n)}\left[a_{1}+b_{1}, \ldots, a_{n}+b_{n} ; \lambda+\mu ; \lambda_{1} x, \ldots, \lambda_{n} x\right],
\end{aligned}
$$

where $\min \{\operatorname{Re}(\lambda), \operatorname{Re}(\mu)\}>0, \max \left\{\left|\lambda_{1} x\right|, \ldots,\left|\lambda_{n} x\right|\right\}<\infty$.

If we set $b_{1}=\cdots=b_{n}=0$ in (2.12), it yields

$$
\begin{array}{r}
\int_{0}^{x} t^{\lambda-1}(x-t)^{\mu-1} \Phi_{2}^{(n)}\left[a_{1}, \ldots, a_{n} ; \lambda ; \lambda_{1} x, \ldots, \lambda_{n} x\right] d t \\
=B(\lambda, \mu) \Phi_{2}^{(n)}\left[a_{1}, \ldots, a_{n} ; \lambda+\mu ; \lambda_{1} x, \ldots, \lambda_{n} x\right]
\end{array}
$$

where $\min \{\operatorname{Re}(\lambda), \operatorname{Re}(\mu)\}>0, \max \left\{\left|\lambda_{1} x\right|, \ldots,\left|\lambda_{n} x\right|\right\}<\infty$.

When $n=1$, we obtain the following result for the generalized Mittag-Leffler functions due to Kilbas et al. [13].

Let $\alpha, \rho, \gamma, \delta, \lambda, \mu, z \in \mathbb{C}, \operatorname{Re}(\rho)>0, \operatorname{Re}(\lambda)>0, \operatorname{Re}(\mu)>0,\left|z x^{\rho}\right|<1$, then

$$
\int_{0}^{x} t^{\lambda-1} E_{\rho, \lambda}^{\gamma}\left(z t^{\rho}\right)(x-t)^{\mu-1} E_{\rho, \mu}^{\delta}\left[z(x-t)^{\rho}\right] d t=x^{\lambda+\mu-1} E_{\rho, \lambda+\mu}^{\gamma+\delta}\left(z x^{\rho}\right) .
$$

Let $\gamma, \mu, \rho, z \in \mathbb{C}, \min \{\operatorname{Re}(\lambda), \operatorname{Re}(\mu)\}>0, \operatorname{Re}(\rho)>0$, then if we set $\delta=1,(2.14)$ reduces to

$$
\int_{0}^{x} x^{\lambda-1} E_{\rho, \lambda}^{\gamma}\left(z x^{\rho}\right)(x-t)^{\mu-1} E_{\rho, \mu}\left[z(x-t)^{\rho}\right]=x^{\lambda+\mu-1} E_{\rho, \lambda+\mu}^{\gamma+1}\left(z x^{\rho}\right)
$$

where $\min \{\operatorname{Re}(\lambda), \operatorname{Re}(\mu)\}>0 ;|\lambda t|<1$. It is interesting to note that by virtue of the identity $[13,(1.8)$, page 379$]$, we arrive at a well-known result [10, (15), page 271]:

$$
\int_{0}^{t} u^{\lambda-1}(t-u)^{\mu-1} \Phi[\gamma, \lambda ; z u) \Phi[\delta, \mu ; z(t-u)] d t=B(\lambda, \mu) t^{\lambda+\mu-1} \Phi(\gamma+\delta, \lambda+\mu ; z t),
$$

where $\min \{\operatorname{Re}(\lambda), \operatorname{Re}(\mu)\}>0$.

Remark 2.2. A detailed and comprehensive account of the multiple Gaussian hypergeometric functions is available from Srivastava and Karlsson [31].

The following result will be found useful in the analysis that follows [19, 21]:

$$
L\left\{{ }_{0} D_{t}^{\alpha} h(t)\right\}=s^{\alpha} H(s)-\left.\sum_{k=0}^{n-1} s^{\alpha-k-1} D_{t}^{\alpha-k-1} h(t)\right|_{t=0} \quad(n-1<\operatorname{Re}(\alpha) \leq n) .
$$




\section{Solution of the generalized fractional integro-differential equation}

Theorem 3.1. Consider the following generalized integro-differential equation of Volterra type:

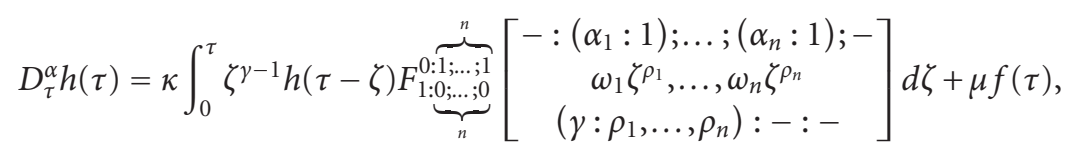

where $0 \leq \tau \leq 1 ; \kappa, \gamma, \mu, \alpha_{j}, \rho_{j}, \omega_{j} \in \mathbb{C} ; \min \{\operatorname{Re}(\alpha), \operatorname{Re}(\gamma)\}>0 ; \operatorname{Re}\left(\rho_{j}\right)>0(j=1, \ldots, n)$, together with the initial conditions

$$
\begin{gathered}
\left.D_{\tau}^{\alpha-k} h(\tau)\right|_{\tau=0}=a_{k} \quad(k=1, \ldots, N), \\
(N:=-[-\operatorname{Re}(\alpha)]: N-1<\operatorname{Re}(\alpha) \leq N ; N \in \mathbb{N}),
\end{gathered}
$$

where $a_{1}, \ldots, a_{N}$ are prescribed constants and $f(\tau)$ is assumed to be continuous on every finite closed interval $[0, T](0<T<\infty)$, and of exponential order $\exp (\eta \tau)$ when $\tau \rightarrow \infty$. Then there exists a unique continuous solution of the Cauchy-type problem (3.1) and (3.2) given by

$$
h(\tau)=\sum_{k=1}^{N} a_{k} \Lambda_{k}(x)+\mu \int_{0}^{\tau} \Theta(\tau-\zeta) f(\zeta) d \zeta
$$

where

$$
\begin{aligned}
& \Lambda_{k}(\tau)=\tau^{\alpha-k} \sum_{r=0}^{\infty} \frac{\kappa^{r} \tau^{(\alpha+\gamma) r}}{\Gamma[\alpha+(\alpha+\gamma) r-k+1]} \\
& \times F_{1: 0 ; \ldots ; 0 ; 0}^{0: \ldots ; 1}\left[\begin{array}{c}
-:\left(\alpha_{1}: 1\right) ; \ldots ;\left(\alpha_{n}: 1\right) ;- \\
\omega_{1} \tau^{\rho_{1}}, \ldots, \omega_{n} \tau^{\rho_{n}}
\end{array}\right] \text {, } \\
& \Theta(\tau)=\tau^{\alpha-1} \sum_{r=0}^{\infty} \frac{\kappa^{r} \tau^{(\alpha+\gamma) r}}{\Gamma[\alpha+(\alpha+\gamma) r]} \cdot F_{1: 0 ; \ldots ; 0}^{0: \overbrace{n} ; \ldots ; 1}\left[\begin{array}{c}
-:\left(\alpha_{1} r: 1\right) ; \ldots ;\left(\alpha_{n} r: 1\right) ;- \\
\omega_{1} \tau^{\rho_{1}}, \ldots, \omega_{n} \tau^{\rho_{n}} \\
\left(\alpha+\alpha r+\gamma r: \rho_{1}, \ldots, \rho_{n}\right):-:-
\end{array}\right] .
\end{aligned}
$$

Proof. Applying the Laplace transform to (3.1) and using (2.17), we find that

$$
s^{\alpha} H(s)-\left.\sum_{k=1}^{N} s^{k-1} D_{\tau}^{\alpha-k} h(\tau)\right|_{\tau=0}=\kappa s^{-\gamma}\left[\prod_{j=1}^{n}\left(1-\frac{\omega_{j}}{s^{\rho_{j}}}\right)^{-\alpha_{j}}\right] H(s)+\mu F(s),
$$

$\min \{\operatorname{Re}(\alpha), \operatorname{Re}(\gamma)\}>0, \operatorname{Re}(s)>0$, where $H(s)$ and $F(s)$ represent, respectively, the Laplace transform of the functions $h(\tau)$ and $f(\tau)$. 
Solving (3.6) under the initial conditions (3.2), we find that

$$
\begin{aligned}
H(s)= & \sum_{k=1}^{N} A_{k} s^{k-\alpha-1}\left[1-\kappa s^{-\alpha} \prod_{j=1}^{n}\left\{s^{-\gamma}\left(1-\frac{\omega_{j}}{s^{\rho_{j}}}\right)^{-\alpha_{j}}\right\}\right]^{-1} \\
& +\mu F(s)\left[1-\kappa s^{-\alpha-\gamma} \prod_{j=1}^{n}\left(1-\frac{\omega_{j}}{s^{\rho_{j}}}\right)^{-\alpha_{j}}\right]^{-1},
\end{aligned}
$$

where it is tacitly assumed that

$$
\left|\kappa s^{-\alpha-\gamma}\left\{\prod_{j=1}^{n}\left(1-\frac{\omega_{j}}{s^{\rho_{j}}}\right)^{-\alpha_{j}}\right\}\right|<1
$$

By the application of the formula (2.1) once again, it is found from (3.7) that

$$
\begin{aligned}
& h(\tau)=\sum_{k=1}^{N} A_{k} \frac{\kappa^{r} \tau^{\alpha+(\alpha+\gamma) r-k}}{\Gamma[\alpha+(\alpha+\gamma) r-k+1]} \\
& \times F_{1: \underbrace{0: 1 ; \ldots ; 0 ; 1}_{n}}^{n}\left[\begin{array}{c}
-:\left(\alpha_{1} r: 1\right) ; \ldots ;\left(\alpha_{n} r: 1\right) ;- \\
\omega_{1} \tau^{\rho_{1}}, \ldots, \omega_{n} \tau^{\rho_{n}} \\
\left(\alpha+\alpha r+\gamma r-k+1: \rho_{1}, \ldots, \rho_{n}\right):-:-
\end{array}\right] \\
& +\sum_{r=0}^{\infty} \kappa^{r} \int_{0}^{\tau} \frac{(\tau-\zeta)^{\alpha+(\alpha+\gamma) r-1}}{\Gamma[\alpha+(\alpha+\gamma) r]} \\
& \times F_{1: \underbrace{0 ; \ldots ; 0 ; 0}_{n}}^{n+\overbrace{1 ; 1}^{n}}\left[\begin{array}{c}
-:\left(\alpha_{1} r: 1\right) ; \ldots ;\left(\alpha_{n} r: 1\right) ;- \\
\omega_{1}(\tau-\zeta)^{\rho_{1}}, \ldots, \omega_{n}(\tau-\zeta)^{\rho_{n}} \\
\left(\alpha r+\gamma r+\alpha: \rho_{1}, \ldots, \rho_{n}\right):-:-
\end{array}\right] f(\zeta) d \zeta .
\end{aligned}
$$

The above expression can be expressed in the form (3.3). This completes the proof of Theorem 3.1.

In order to establish the uniqueness of the solution (3.3), we set $\zeta \rightarrow \tau-\zeta$ in (3.1) and operate upon both sides by $D_{\tau}^{-\alpha}(\operatorname{Re}(\alpha)>0)$. Next, if we apply the Dirichlet formula [25] and make use of the Eulerian integral for the Beta function, we arrive at the following equation:

$$
h(\tau)=\mu D_{\tau}^{-\alpha} f(\tau)+\kappa \int_{0}^{\tau} h(\varsigma)(\tau-\varsigma)^{\alpha+\gamma-1} F_{1: 0 ; \ldots ; 0}^{0: \overbrace{1 ; \ldots ;}^{n}}\left[\begin{array}{c}
-:\left(\alpha_{1}: 1\right) ; \ldots ;\left(\alpha_{n}: 1\right) ;- \\
\omega_{1}(\tau-\varsigma)^{\rho_{1}}, \ldots, \omega_{n}(\tau-\varsigma)^{\rho_{n}} \\
\left(\alpha+\gamma: \rho_{1}, \ldots, \rho_{n}\right):-:-
\end{array}\right] d \varsigma .
$$

Since (3.10) is a Volterra integral equation with a continuous kernel, it does admit a unique continuous solution (see [15]).

Remark 3.2. The solution of the Cauchy-type problem (3.1) and (3.2) can also be developed by the method of successive approximations. In this connection, see [13,23]. 


\section{Special cases of Theorem 3.1}

If we set

$$
\rho_{1}=\cdots=\rho_{n}=1 \text {, }
$$

then by virtue of the identity (2.10), we arrive at the following result recently obtained by Srivastava and Saxena [33].

COROLLARY 4.1. Under the various relevant hypotheses of Theorem 3.1, a unique continuous solution of the Cauchy-type problem involving the Volterra-type integro-differential equation

$$
D_{\tau}^{\alpha} h(\tau)=\frac{\kappa}{\Gamma(\gamma)} \int_{0}^{\tau} \zeta^{\gamma-1} h(\tau-\zeta) \Phi_{2}^{(n)}\left[\alpha_{1}, \ldots, \alpha_{n}: \gamma ; \omega_{1} \zeta, \ldots, \omega_{n} \zeta\right] d t+\mu f(\tau)
$$

where $0<\tau \leq 1, \alpha_{j}, \kappa, \gamma, \mu \in \mathbb{C}, \min \{\operatorname{Re}(\alpha), \operatorname{Re}(\gamma)\}>0$, together with the initial conditions (3.2), is given by

$$
h(\tau)=\sum_{k=1}^{N} a_{k} \Omega_{k}(\tau)+\mu \int_{0}^{\tau} \Xi(\tau-t) f(t) d \zeta,
$$

where

$$
\begin{aligned}
& \Omega_{k}(\tau):=\tau^{\alpha-k} \sum_{r=0}^{\infty} \frac{\kappa^{r} \tau^{(\alpha+\gamma) r}}{\Gamma[(\alpha+\gamma) r+\alpha-k+1]} \\
& \times \Phi_{2}^{(n)}\left[\alpha_{1} r, \ldots, \alpha_{n} r ;(\alpha+\gamma) r+\alpha-k+1 ; \omega_{1} \tau, \ldots, \omega_{m} \tau\right] \quad(k=1, \ldots, N), \\
& \Xi(\tau)=\tau^{\alpha-1} \sum_{r=0}^{\infty} \frac{\kappa^{r} \tau^{(\alpha+\gamma) r}}{\Gamma[\alpha+(\alpha+\gamma) r]} \cdot \Phi_{2}^{(n)}\left[\alpha_{1} r, \ldots, \alpha_{n} r ; \alpha+(\alpha+\gamma) r ; \omega_{1} \tau, \ldots, \omega_{n} \tau\right] .
\end{aligned}
$$

If we take $n=1$, then Theorem 3.1 reduces to the following result given by Kilbas et al. [13].

COROLLARY 4.2. Under the various relevant hypotheses of Theorem 3.1, a unique continuous solution of the Cauchy-type problem involving the Volterra-type integro-differential equation

$$
D_{\tau}^{\alpha} h(\tau)=\kappa \int_{0}^{x} \zeta^{\mu-1} h(\tau-\zeta) E_{\rho, \mu}^{\gamma}\left[\omega \zeta^{\rho}\right] d \zeta+f(\tau)
$$

$0 \leq \tau \leq 1 ; \kappa, \gamma, \rho, \omega, \mu \in \mathbb{C} ; \min \{\operatorname{Re}(\alpha), \operatorname{Re}(\gamma), \operatorname{Re}(\omega), \operatorname{Re}(\mu)\}>0$, together with the initial conditions (3.2), is given by

$$
h(\tau)=\sum_{k=1}^{N} a_{k} \Psi_{k}(\tau)+\int_{0}^{\tau} \Theta_{1}(\tau-\zeta) f(\zeta) d \zeta,
$$


where

$$
\begin{gathered}
\Psi_{k}(\zeta):=\tau^{\alpha-k} \sum_{r=0}^{\infty} \kappa^{r} \tau^{(\alpha+\mu) r} E_{\rho, \alpha+(\alpha+\mu) r-k+1}^{\gamma r}\left(\omega \tau^{\rho}\right) \quad(k=1, \ldots, N), \\
\Theta_{1}(\tau):=x^{\alpha-1} \sum_{r=0}^{\infty} \kappa^{r} \tau^{(\alpha+\mu) r} E_{\rho, \alpha+(\alpha+\mu) r}^{\gamma r}\left(\omega \tau^{\rho}\right) .
\end{gathered}
$$

If we set $\rho=1$ and use the identity [13, (1.8), page 379], Corollary 4.2 reduces to the following result given by Kilbas et al. [13, (4.8), page 391].

COROLlary 4.3. Under the various relevant hypotheses of Theorem 3.1, a unique continuous solution of the Cauchy-type problem involving the Volterra-type integro-differential equation

$$
D_{\tau}^{\alpha} h(\tau)=\frac{\kappa}{\Gamma(\gamma)} \int_{0}^{\tau} \zeta^{\gamma-1} h(\tau-\zeta){ }_{1} F_{1}(\beta ; \gamma ; \lambda \zeta) d \zeta+\mu f(\tau)
$$

$(0 \leq \tau \leq 1, \beta, \gamma, \kappa, \mu \in \mathbb{C}, \min \{\operatorname{Re}(\alpha), \operatorname{Re}(\gamma)\}>0)$, together with the initial conditions (3.2), is given by

$$
h(\tau)=\sum_{k=1}^{N} a_{k} \vartheta_{k}(\tau)+\mu \int_{0}^{\tau} \theta(\tau-\zeta) f(\zeta) d \zeta
$$

where

$$
\begin{gathered}
\vartheta_{k}(\tau):=\tau^{\alpha-k} \sum_{r=0}^{\infty} \frac{\kappa^{r} \tau^{(\alpha+\gamma) r}}{\Gamma(\alpha+(\alpha+\gamma) r-k+1)}{ }_{1} F_{1}(\beta r ; \alpha+(\alpha+\gamma) r-k+1 ; \lambda \tau) \quad(k=1, \ldots, N), \\
\theta(\tau):=\tau^{\alpha-1} \sum_{r=0}^{\infty} \frac{\kappa^{r} \tau^{(\alpha+\gamma) r}}{\Gamma(\alpha+(\alpha+\gamma) r)}{ }_{1} F_{1}(\beta r ; \alpha+(\alpha+\gamma) r ; \lambda \tau) .
\end{gathered}
$$

Remark 4.4. In its further special case, when

$$
f(\tau)=\frac{1}{\Gamma(\delta)} \tau_{1}^{\delta-1} F_{1}(\rho ; \delta ; \omega \tau)
$$

then if we apply the integral addition formula (2.17), Corollary 4.3 would yield the main result of Saxena and Kalla [26, Theorem 1, page 91], which itself is a generalization of the result given earlier by Al-Shammery et al. [2, (14), page 504].

Next, if we set

$$
f(\tau)=\tau^{\nu-1} F_{1: \underbrace{0: 1 ; \ldots, 0}_{n}}^{n}\left[\begin{array}{c}
-:\left(\delta_{1}: 1\right) ; \ldots ;\left(\delta_{n}: 1\right) ;- \\
\omega_{1} \tau^{\rho_{1}}, \ldots, \omega_{n} \tau^{\rho_{n}} \\
-:\left(\nu: \rho_{1}, \ldots, \rho_{n}\right):-:-
\end{array}\right],
$$

and apply the general (multivariable) integral addition formula (2.9), then Theorem 3.1 gives us the following result. 
COROLLARY 4.5. Under the various relevant hypotheses of Theorem 3.1, a unique continuous solution of the Cauchy-type problem involving the Volterra-type integro-differential equation

$$
\begin{aligned}
& D_{\tau}^{\alpha} h(x)=\kappa \int_{0}^{\tau} \zeta^{\gamma-1} h(\tau-\zeta) F_{1: \underbrace{0: 1 ; \ldots ; 0}_{n}}^{\overbrace{0}^{n}}\left[\begin{array}{c}
-:\left(\alpha_{1}: 1\right) ; \ldots ;\left(\alpha_{n}: 1\right) ;- \\
\omega_{1} \zeta^{\rho_{1}}, \ldots, \omega_{n} \zeta^{\rho_{n}} \\
\left(\gamma: \rho_{1}, \ldots, \rho_{n}\right):-:-
\end{array}\right] d \zeta \\
& +\mu \tau^{\nu-1} F_{1: 0 ; \ldots ; 0}^{0: 1 ; \ldots ; 0} \underbrace{n}_{n}\left[\begin{array}{c}
-:\left(\delta_{1}, 1\right) ; \ldots ;\left(\delta_{n}, 1\right) ;- \\
\omega_{1} \tau^{\rho_{1}}, \ldots, \omega_{n} \tau^{\rho_{n}} \\
\left(\nu: \rho_{1}, \ldots, \rho_{n}\right):-:-
\end{array}\right]
\end{aligned}
$$

$\left(0 \leq \tau \leq 1 ; \kappa, \gamma, \mu, \nu, \alpha_{j}, \delta_{j}, \omega_{j}, \rho_{j} \in \mathbb{C} ; \min \{\operatorname{Re}(\alpha), \operatorname{Re}(\gamma), \operatorname{Re}(\nu)\}>0\right), \operatorname{Re}\left(\rho_{j}\right)>0 \quad(j=$ $1, \ldots, n)$, with the initial conditions (3.2) is given by

$$
h(\tau):=\sum_{k=1}^{N} A_{k} \Lambda_{k}(\tau)+\mu \Delta(\tau)
$$

where

$$
\Delta(\tau):=\tau^{\alpha+\nu-1} \sum_{r=0}^{\infty} \frac{\kappa^{r} \tau^{(\alpha+\gamma) r}}{\Gamma[\alpha+(\alpha+\gamma) r]} F_{1: \underbrace{0 ; \ldots ; 0}_{n}}^{0: 1 ; \ldots ;}{ }^{n}\left[\begin{array}{c}
-:\left(\alpha_{1} r+\delta_{1}: 1\right) ; \ldots ;\left(\alpha_{n} r+\delta_{n}: 1\right) ;- \\
\omega_{1} \tau^{\rho_{1}}, \ldots, \omega_{n} \tau^{\rho_{n}} \\
\left(\alpha r+\gamma r+\alpha+\nu: \rho_{1}, \ldots, \rho_{n}\right):-:-
\end{array}\right],
$$

and $\Lambda_{k}$ 's are given in (3.4).

\section{A Cauchy-type problem involving the Caputo fractional derivatives}

In connection with certain investigations, especially in the theory of viscoelasticity and hereditary solid mechanics, Caputo introduced the following definition for the fractional derivative of order $\alpha>0$ of a casual function $f(t)$ (i.e., $f(t)=0$, for $t<0$ ), which arose in several important earlier works (see, for details [21, page 78]):

$$
\begin{aligned}
\frac{d^{\alpha}}{d t^{\alpha}} h(t) & :=h^{(m)}(t) \quad\left(\alpha=m \in \mathbb{N}_{0}:=\mathbb{N} \cup\{0\}\right) \\
& =\frac{1}{\Gamma(m-\alpha)} \int_{0}^{t} \frac{h^{(m)}(\tau)}{(t-\tau)^{\alpha-m+1}} d \tau \quad(m-1<\alpha<m ; m \in \mathbb{N}),
\end{aligned}
$$

where $h^{(m)}(t)$ denotes the usual (ordinary) derivative of $h(t)$ of order $m\left(m \in \mathbb{N}_{0}\right)$. It readily follows from the definitions (1.5) and (5.1) that

$$
L\left\{\frac{d^{\alpha}}{d t^{\alpha}} h(t): s\right\}=s^{\alpha} H(s)-\sum_{k=0}^{m-1} s^{\alpha-k-1} h^{(k)}(0) \quad(m-1<\alpha \leq m ; m \in \mathbb{N}),
$$

which is preferred for initial-value problems of physical sciences than (2.17), $H(s)$ is given by (1.5).

The method of proof of Theorem 3.1 can now be applied mutatis mutandis in order to solve the following Cauchy problem involving Caputo derivatives defined by (5.1). 
THEOREM 5.1. Consider the following generalized fractional integro-differential equation of Volterra type

$$
\frac{d^{\alpha}}{d \tau^{\alpha}} h(\tau)=\kappa \int_{0}^{\tau} \zeta^{\gamma-1} h(\tau-\zeta) F_{1: 0 ; \ldots ; 0}^{0: \overbrace{1 ; \ldots ;}^{n}}\left[\begin{array}{c}
-:\left(\alpha_{1}: 1\right) ; \ldots ;\left(\alpha_{n}: 1\right) ;- \\
\omega_{1} \zeta^{\rho_{1}}, \ldots, \omega_{n} \zeta^{\rho_{n}} \\
\left(\gamma: \rho_{1}, \ldots, \rho_{n}\right):-:-
\end{array}\right] d \zeta+\mu f(\tau),
$$

where $0 \leq \tau \leq 1 ; \kappa, \mu, \alpha_{j}, \rho_{j}, \omega_{j} \in \mathbb{C} ; \min \{\operatorname{Re}(\alpha), \operatorname{Re}(\gamma)\}>0 ; \operatorname{Re}\left(\rho_{j}\right)>0(j=1, \ldots, n)$, together with the initial conditions

$$
\left.\frac{d^{k}}{d \tau^{k}} h(\tau)\right|_{\tau=0}=b_{k} \quad(k=1, \ldots, N)
$$

$(N:=-[-\operatorname{Re}(\alpha)] ; N-1<\operatorname{Re}(\alpha) \leq N ; N \in \mathbb{N})$, where $b_{1}, \ldots, b_{N}$ are prescribed constants and $f(\tau)$ is constrained as in Theorem 3.1. Then there exists a unique continuous solution of the Cauchy-type problem (5.4) and (5.5) given by

$$
h(\tau)=\sum_{k=1}^{N} b_{k} \Lambda_{k}(x)+\mu \int_{0}^{\tau} \Theta(\tau-\zeta) f(\zeta) d \zeta
$$

where $\Lambda_{k}(\tau)(k=1, \ldots, N)$ and $\Theta(t)$ are already defined by (3.4) and (3.5), respectively.

Clearly, the assertions of Theorems 3.1 and 5.1 would coincide when we set

$$
\alpha=N \quad(N \in \mathbb{N})
$$

More interestingly, it is fairly straightforward to apply Theorem 5.1 in order to deduce analogues of Corollaries 4.1 and 4.2 for the corresponding Cauchy problems involving the Caputo fractional derivatives defined by (5.1). For example, by taking $\rho_{1}=\cdots=\rho_{n}=1$, it yields the following analogues of Corollary 4.1 dealing with a more general Cauchy problem than that associated with FEL equation (1.1).

COROLlary 5.2. Under the various relevant hypotheses of Theorem 3.1, a unique continuous solution of the Cauchy-type problem involving the Volterra-type integro-differential equation

$$
D_{\tau}^{\alpha} h(\tau)=\frac{\kappa}{\Gamma(\gamma)} \int_{0}^{\tau} t^{\gamma-1} h(\tau-\zeta) \Phi_{2}^{(n)}\left[\alpha_{1}, \ldots, \alpha_{n}: \gamma ; \omega_{1} \zeta, \ldots, \omega_{n} \zeta\right] d t+f(\tau)
$$

where $0 \leq \tau \leq 1, \gamma, \alpha_{j}, \kappa \in \mathbb{C}, \min \{\operatorname{Re}(\alpha), \operatorname{Re}(\gamma)\}>0$, together with the initial conditions (3.2) is given by

$$
h(\tau)=\sum_{k=1}^{N} b_{k} \Omega_{k}(\tau)+\int_{0}^{\tau} \Xi(\tau-t) f(t) d t
$$

where $\Omega_{k}(\tau)(k=1, \ldots, n)$ and $\Xi(\tau)$ are defined by (4.4) and (4.5), respectively. 


\section{Solution of the generalized Volterra integral equation}

In this section, we present a generalization of the Volterra integral equation quite recently given by Srivastava and Saxena [33].

Theorem 6.1. The Volterra-type integral equation

$$
D_{\tau}^{-\nu} h(\tau)=\kappa \int_{0}^{\tau}(\tau-\zeta)^{\gamma-1} h(\zeta) F_{1: \underbrace{0: \ldots ; \ldots ; 0}_{n}}^{\overbrace{1 ; \ldots ;}^{n}}\left[\begin{array}{c}
-:\left(\alpha_{1}: 1\right) ; \ldots ;\left(\alpha_{n}: 1\right) ;- \\
\omega_{1}(\tau-\zeta)^{\rho_{1}}, \ldots, \omega_{n}(\tau-\zeta)^{\rho_{n}} \\
\left(\gamma: \rho_{1}, \ldots, \rho_{n}\right):-:-
\end{array}\right] d \zeta+\mu f(\tau)
$$

has its solution given explicitly by

$$
\begin{aligned}
& h(\tau)=-\mu \sum_{r=0}^{\infty} \kappa^{-r-1} \int_{0}^{\tau}(\tau-\zeta)^{m+\nu r-\gamma r-\gamma-1}\left(\frac{d^{m}}{d \zeta^{m}} f(\zeta)\right)
\end{aligned}
$$

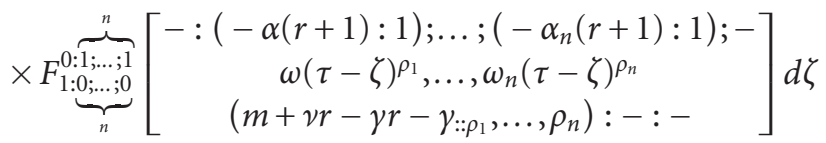

$\left(0 \leq \operatorname{Re}(\gamma)<\min \{m, \nu\} ; f \in \mathbb{C}^{m}[0, \infty) ; f^{(j)}(0)=0(j=0,1, \ldots,(m-1) ; m \in \mathbb{N} ; \kappa, \mu \in \mathbb{C} ;\right.$ $\left.\nu \geq 0) \cdot \operatorname{Re}\left(\rho_{j}\right)>0\right)$.

Proof. In view of the results (1.4) and (2.1), if we apply the Laplace transform operator on both sides of Volterra integral equation (6.1), it readily follows that

$$
H(s)=\mu F(s)\left[s^{-\gamma}-\kappa s^{-\gamma} \prod_{j=1}^{n}\left\{\left(1-\frac{\omega_{j}}{s^{\rho_{j}}}\right)^{-\alpha_{j}}\right\}^{-1}\right]
$$

$\nu \geq 0 ; \operatorname{Re}(\gamma)>0, \operatorname{Re}(s)>\max \left\{0, \operatorname{Re}\left(\omega_{j}\right), \operatorname{Re}(\eta)\right\}, \max _{1 \leq j \leq n}\left|\omega_{j} / s^{\rho_{j}}\right|<1, \operatorname{Re}\left(\rho_{j}\right)>0(j=$ $1, \ldots, n)$, where $F(s)$ and $H(s)$ denote the Laplace transforms of $f(\tau)$ and $h(\tau)$, respectively.

Now, if we assume that

$$
\left|\kappa^{-1} s^{-\gamma}-\kappa s^{-\gamma} \prod_{j=1}^{n}\left\{\left(1-\frac{\omega_{j}}{s^{\rho_{j}}}\right)^{-\alpha_{j}}\right\}^{-1}\right|<1
$$

it is found from (6.3) that

$$
\begin{gathered}
H(s)=-\mu \sum_{r=0}^{\infty} \kappa^{-r-1} s^{\gamma-m-(\nu-\gamma) r} \prod_{j=1}^{m}\left\{\left(1-\frac{\omega_{j}}{s^{\rho_{j}}}\right)^{\alpha_{j}(r+1)}\right\}\left[s^{m} F(s)\right] \\
(0<\operatorname{Re}(\gamma)<\min \{m, \nu\} ; m \in \mathbb{N} ; \nu \geq 0),
\end{gathered}
$$

which leads us easily to the desired result (6.2) under the conditions stated along with Theorem 6.1. 
When $\rho_{1}=\cdots=\rho_{n}=1$, then using the relation (2.12), it immediately yields the following result recently given by Srivastava and Saxena [33], which itself is a generalization of Srivastava's result [27].

Corollary 6.2. The Volterra-type integral equation

$$
\begin{aligned}
D_{\tau}^{-\gamma} h(\tau)= & \kappa \int_{0}^{\tau} \frac{(\tau-\zeta)^{\gamma=1}}{\Gamma(\gamma)} h(\zeta) \\
& \cdot \Phi_{2}^{(n)}\left[\alpha_{1}, \ldots, \alpha_{n} ; \gamma ; \omega_{1}(\tau-\zeta), \ldots, \omega_{n}(\tau-\zeta)\right] d \zeta+\mu f(\tau)
\end{aligned}
$$

has its solution given explicitly by

$$
\begin{aligned}
h(\tau)= & -\mu \sum_{r=0}^{\infty} \kappa^{-r-1} \int_{0}^{\tau} \frac{(\tau-\zeta)^{m+(\nu-\gamma) r-\gamma-1}}{\Gamma(m+(\nu-\gamma) r-\gamma)}\left(\frac{d^{m}}{d \zeta^{m}} f(\zeta)\right) \\
& \cdot \Phi_{2}^{(n)}\left[-\alpha_{1}(r+1), \ldots,-\alpha_{n}(r+1) ; m+(\nu-\gamma) r-\gamma ; \omega_{1}(\tau-\zeta), \ldots, \omega_{n}(\tau-\zeta)\right] d \zeta
\end{aligned}
$$

$\left(0<\operatorname{Re}(\gamma)<\min \{m, \nu\} ; f \in \mathbb{C}^{m}[0, \infty) ; f^{(j)}(0)=0(j=0,1, \ldots, m-1) ; m \in N ; \kappa, \mu \in \mathbb{C} ;\right.$ $\nu \geq 0)$.

\section{Acknowledgment}

This research is supported, in part, by a grant from the University Grants Commission of India.

\section{References}

[1] M. Abramowitz and I. A. Stegun, eds., Handbook of Mathematical Functions with Formulas, Graphs and Mathematical Tables, Applied Mathematics Series, vol. 55, National Bureau of Standards, District of Columbia, 1968.

[2] A. H. Al-Shammery, S. L. Kalla, and H. G. Khajah, A fractional generalization of the free electron laser equation, Fract. Calc. Appl. Anal. 2 (1999), no. 4, 501-508.

[3] - On a generalized fractional integro-differential equation of Volterra-type, Integral Transform. Spec. Funct. 9 (2000), no. 2, 81-90.

[4] L. Boyadjiev and S. L. Kalla, Series representations of analytic functions and applications, Fract. Calc. Appl. Anal. 4 (2001), no. 3, 379-408.

[5] L. Boyadjiev, S. L. Kalla, and H. G. Khajah, Analytical and numerical treatment of a fractional integro-differential equation of Volterra type, Math. Comput. Modelling 25 (1997), no. 12, $1-9$.

[6] M. Caputo, Linear model of dissipation whose Q is almost frequency independent, II, Geophysics. J. R. Astr. Soc. 13 (1967), 529-539.

[7] G. Dattoli, L. Giannessi, L. Mezi, A. Torre, and R. Caloi, FEL time-evolution operator, Nuclear. Instr. Methods A 304 (1991), 541-544.

[8] G. Dattoli, S. Lorenzutta, G. Maino, D. Tocci, and A. Torre, Results for an integro-differential equation arising in a radiation evaluation problem, Proceedings of the International Workshop on Dynamics of Transport in Plasmas and Charged Beams (Torino, Italy, July 1994), World Scientific, Singapore, 1995, pp. 202-221.

[9] G. Dattoli, S. Lorenzutta, G. Maino, and A. Torre, Analytical treatment of the high-gain free electron laser equation, Radiat. Phys. Chem. 48 (1996), 29-40. 
[10] A. Erdélyi, W. Magnus, F. Oberhettinger, and F. G. Tricomi, Higher Transcendental Functions. Vols. I, II, McGraw-Hill Book, New York, 1953.

[11] , Tables of Integral Transforms. Vol. I, McGraw-Hill Book, New York, 1954.

[12] R. Hilfer, ed., Applications of Fractional Calculus in Physics, World Scientific, New Jersey, 2000.

[13] A. A. Kilbas, M. Saigo, and R. K. Saxena, Solution of Volterra integrodifferential equations with generalized Mittag-Leffler function in the kernels, J. Integral Equations Appl. 14 (2002), no. 4, 377-396.

[14]_- Generalized Mittag-Leffler function and generalized fractional calculus operators, Integral Transform. Spec. Funct. 15 (2004), no. 1, 31-49.

[15] M. L. Krasnov, A. I. Kiselev, and G. L. Makarenko, Integral Equations, 3rd ed., Mir Publishers, Moscow, 1983.

[16] F. Mainardi and M. Tomirotti, Seismic pulse propagation with constant $Q$ and stable probability distributions, Ann. Geophysica 40 (1997), no. 5, 1311-1328.

[17] A. M. Mathai and R. K. Saxena, Various practical problems in probability and statistics where Lauricella's confluent hypergeometric function appears naturally, Ganita Sandesh 1 (1987), no. 1-2, 41-48.

[18] R. Metzler and J. Klafter, The random walk's guide to anomalous diffusion: a fractional dynamics approach, Phys. Rep. 339 (2000), no. 1, 1-77.

[19] K. S. Miller and B. Ross, An Introduction to the Fractional Calculus and Fractional Differential Equations, A Wiley-Interscience Publication, John Wiley \& Sons, New York, 1993.

[20] K. B. Oldham and J. Spanier, The Fractional Calculus. Theory and Applications of Differentiation and Integration to Arbitrary Order, Mathematics in Science and Engineering, vol. 111, Academic Press, New York, 1974.

[21] I. Podlubny, Fractional Differential Equations. An Introduction to Fractional Derivatives, Fractional Differential Equations, to Methods of Their Solution and Some of Their Applications, Mathematics in Science and Engineering, vol. 198, Academic Press, California, 1999.

[22] T. R. Prabhakar, A singular integral equation with a generalized Mittag Leffler function in the kernel, Yokohama Math. J. 19 (1971), 7-15.

[23] L. B. Rall, Computational Solution of Nonlinear Operator Equations, Robert E. Krieger Publishing, New York, 1979, corrected reprint of the 1969 original (John Wiley \& Sons, New York, 1969).

[24] M. Saigo and R. K. Saxena, Unified fractional integral formulas for the multivariable H-function. III, J. Fract. Calc. 20 (2001), 45-68.

[25] S. G. Samko, A. A. Kilbas, and O. I. Marichev, Fractional Integrals and Derivatives. Theory and Applications, Gordon and Breach Science Publishers, Yverdon, 1993.

[26] R. K. Saxena and S. L. Kalla, On a fractional generalization of the free electron laser equation, Appl. Math. Comput. 143 (2003), no. 1, 89-97.

[27] H. M. Srivastava, An integral equation involving the confluent hypergeometric function of several complex variables, Appl. Anal. 5 (1976), no. 4, 251-256.

[28] H. M. Srivastava and R. G. Buschman, Theory and Applications of Convolution Integral Equations, Mathematics and Its Applications, vol. 79, Kluwer Academic, Dordrecht, 1992.

[29] H. M. Srivastava and M. C. Daoust, Certain generalized Neumann expansions associated with the Kampé de Fériet function, Nederl. Akad. Wetensch. Proc. Ser. A 72 = Indag. Math. 31 (1969), 449-457.

[30] - A note on the convergence of Kampé de Fériet's double hypergeometric series, Math. Nachr. 53 (1972), 151-159.

[31] H. M. Srivastava and P. W. Karlsson, Multiple Gaussian Hypergeometric Series, Ellis Horwood Series: Mathematics and Its Applications, Ellis Horwood, Chichester, 1985, John Wiley \& Sons, New York. 


\section{Solution of Volterra-type equations}

[32] H. M. Srivastava and R. K. Saxena, Operators of fractional integration and their applications, Appl. Math. Comput. 118 (2001), no. 1, 1-52.

[33] Some Volterra-type fractional integro-differential equations with a multivariable confluent hypergeometric function in their kernel, to appear in the J. of Integral Equations Appl.

R. K. Saxena: Department of Mathematics and Statistics, Jai Narain Vyas University, Jodhpur 342004, India

E-mail address: ravisax@sancharnet.in

S. L. Kalla: Department of Mathematics and Computer Science, Kuwait University, P.O. Box 5969, Safat 13060, Kuwait

E-mail address: kalla@mcs.sci.kuniv.edu.kw 


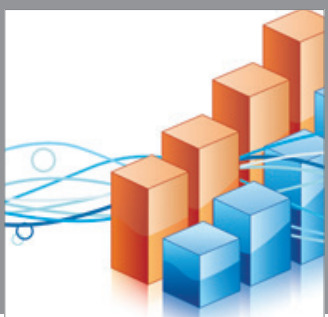

Advances in

Operations Research

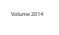

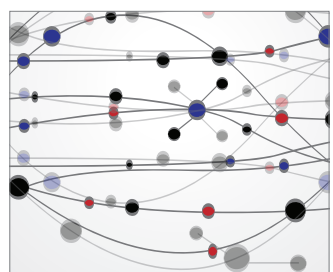

\section{The Scientific} World Journal
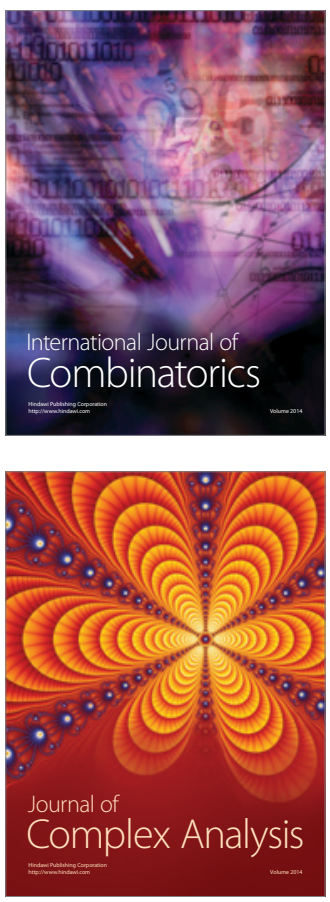

International Journal of

Mathematics and

Mathematical

Sciences
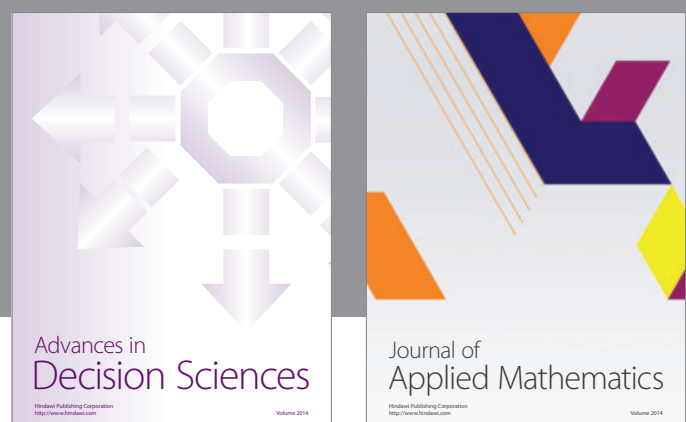

Journal of

Applied Mathematics
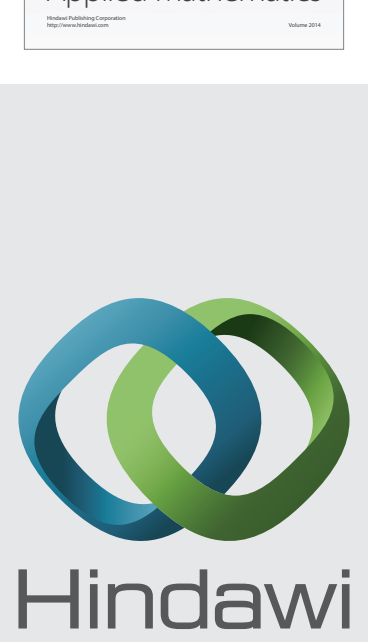

Submit your manuscripts at http://www.hindawi.com
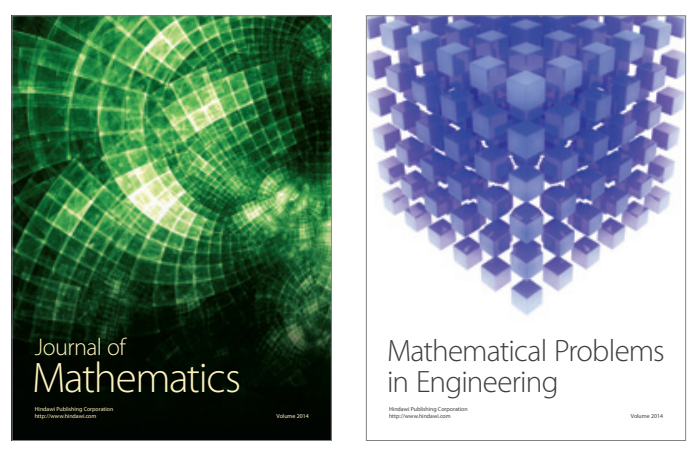

Mathematical Problems in Engineering
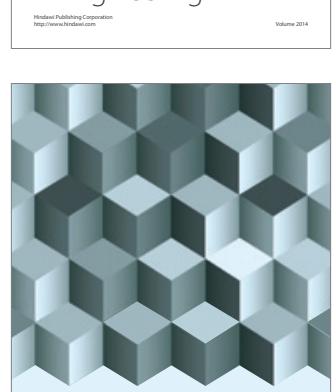

Journal of

Function Spaces
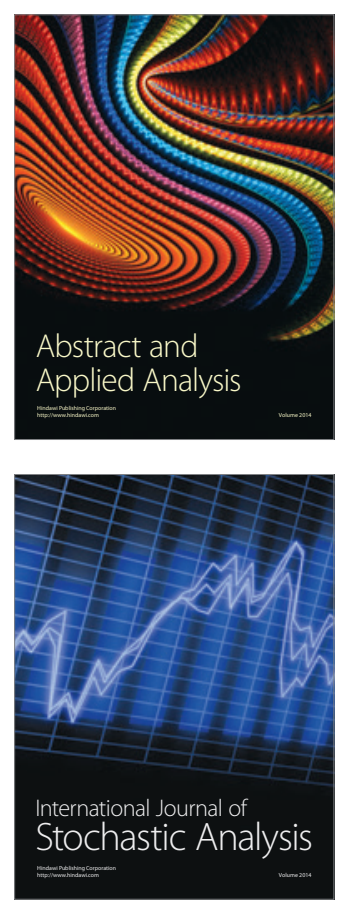

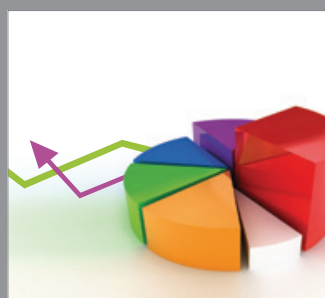

ournal of

Probability and Statistics

Promensencen
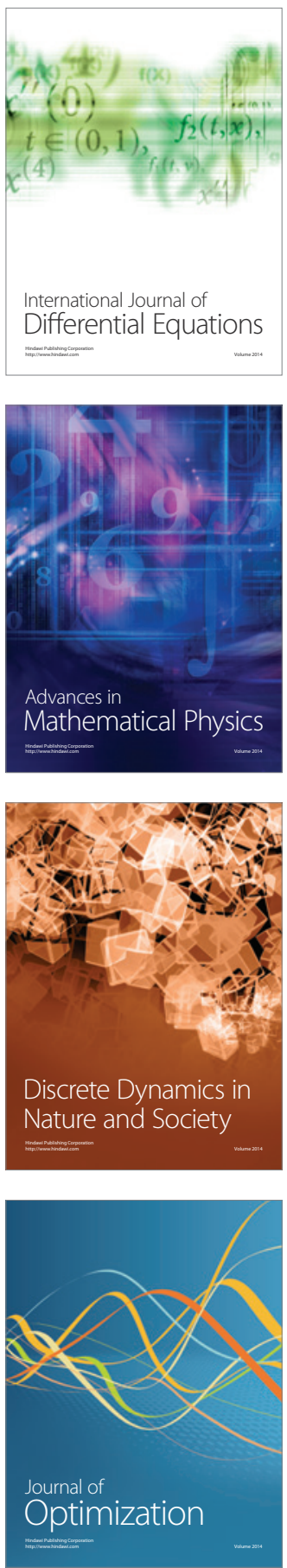